Gynäkologe $2011 \cdot 44: 954-955$

DOI 10.1007/s00129-011-2818-3

Online publiziert: 20. November 2011

(c) Springer-Verlag 2011

\author{
E. Schleußner \\ Abteilung Geburtshilfe, Universitätsklinikum Jena, Friedrich-Schiller-Universität, Jena
}

\title{
Neurologische und psychiatrische Erkrankungen in der Schwangerschaft
}

erhöhte Inzidenz aufweisen (insbesondere sei auf die Depression post partum verwiesen) und

- schwangerschaftsspezifische Erkrankungen, die mit neurologischen Symptomen einhergehen (zentral ist dabei die schwere Präeklampsie/Eklampsie). mögliche teratogene Effekte einer medikamentösen Therapie bestehen viele Unsicherheiten und Ängste bei den betroffenen Frauen wie auch bei ihren betreuenden Ärzten. Dies spiegelt sich wider in einem hohen Beratungsbedarf. Nicht selten kommt es zu fatalen Fehlentscheidungen, wie überstürztem Abbruch notwendiger medikamentöser Therapien bis hin zu Schwangerschaftsabbrüchen aus ungerechtfertigten Fehlbildungsängsten. Solche Risikoschwangerschaften sollten deshalb gut geplant und eng interdisziplinär betreut werden.

Grundsätzlich kann jede neurologische und seelische Erkrankung auch während der Schwangerschaft oder im Wochenbett auftreten. Die dabei zu beachtenden Besonderheiten der Therapie müssen in enger interdisziplinärer Abstimmung zwischen den betreuenden Frauenärzten, Neurologen oder Psychiatern erfolgen.

Die Erkrankungen in der Schwangerschaft lassen sich in drei Gruppen einteilen [1]:

- Erkrankungen, die vorher bestehen oder sich zufällig erst während der Schwangerschaft oder im Wochenbett manifestieren - allen voran die Epilepsie, aber auch Migräne oder multiple Sklerose,

- Erkrankungen, die auch außerhalb der Schwangerschaft auftreten, aber im Umfeld der Schwangerschaft eine
Die neurologischen Komplikationen hypertensiver Schwangerschaftserkrankungen sind wiederholt in Der Gynäkologe thematisiert worden [2], sodass sie in diesem Themenheft nicht erneut dargestellt werden.

Die Beiträge dieses Heftes gehen auf das 12. Jenaer Geburtshilfesymposium 2010 zurück, das von den Universitätskliniken für Geburtshilfe und Neurologie gemeinsam organisiert wurde, um einen praxisorientierten Überblick über die aktuellen Konzepte in Betreuung und Therapie der wichtigsten neurologischen und psychiatrischen Krankheiten während der Schwangerschaft zu geben.

Die häufigste neurologische Erkrankung bei Frauen im reproduktiven Alter ist die Epilepsie, ihre medikamentöse Therapie weist wesentliche teratogene $\mathrm{Ri}$ siken auf. In dem von Uwe Schneider (Geburtshelfer) und Albrecht Kunze (Neurologe), einem interdisziplinären Autorenteam, verfassten Beitrag werden ausgehend von der präkonzeptionellen $\mathrm{Be}$ ratung die Behandlung während der Schwangerschaft, die Betreuung unter der Geburt sowie die Besonderheiten im Wochenbett und während der Stillperiode ausführlich dargestellt. Neben den Empfehlungen für die antiepileptische Therapie werden die krankheitsspezifischen
Risiken für Schwangerschafts- und Geburtskomplikationen sowie insbesondere das Vorgehen bei einem epileptischen Krampfanfall, besprochen.

Speziell mit den teratogenen Risiken antiepileptischer Therapien befasst sich der Beitrag von Philine Senf und Bettina Schmitz von der EURAP Study Group Deutschland. Das European Registry of Antiepileptic drugs in Pregnancy ist die weltweit größte Datensammlung zum Verlauf von Schwangerschaften unter Antiepileptikatherapie. Auf der Basis der Auswertung vom 7555 Schwangerschaften (Stand 05/2011) werden die am häufigsten verwendeten Medikamente hinsichtlich ihres teratogenen Potenzials bewertet und Empfehlungen für die Praxis gegeben.

Auf die wohl häufigste neurologische Erkrankung junger Frauen gehen Ingo W. Husstedt und Stefan Evers in ihrem Beitrag ein. Etwa 25\% aller Frauen im reproduktiven Alter leiden an Migräne, die Probleme nehmen während der Schwangerschaft häufig ab. Bei mehr als der Hälfte der Patientinnen treten jedoch bereits in der ersten Woche post partum erneute Migräneattacken auf. Die Autoren geben Hinweise zur Akuttherapie des Migräneanfalls und gehen ausführlich auf die Besonderheiten der Pharmakotherapie während der Schwangerschaft und Stillzeit ein.

Die multiple Sklerose ist eine besonders schwerwiegende Erkrankung junger Frauen, auf die im Artikel von Matthias Schwab eingegangen wird. Wie bei anderen Autoimmunerkrankungen nimmt die Krankheitsaktivität während der Schwangerschaft häufig ab, doch postpartal steigt das Risiko für einen Erkrankungsschub stark an. Einer geeigneten Schubprophy- 
laxe kommt also große Bedeutung zu. Sowohl die Behandlung als auch die Prophylaxe eines Krankheitsschubes wird mit klaren Empfehlungen für das praktische Vorgehen dargestellt.

Der Beitrag von Sandy Berger und Karl-Jürgen Bär ist seelischen Erkrankungen in der Schwangerschaft gewidmet. Während einer Schwangerschaft existiert kein Schutz vor psychiatrischen Erkrankungen, auch Suizide und Suizidversuche werden von schwangeren Frauen durchgeführt. Depressive Episoden sowie bipolare Störungen sind dabei sehr häufig, aber auch schizophrene Psychosen und Zwangserkrankungen können auftreten. Es werden die heute etablierten Therapieansätze dargestellt sowie die Besonderheiten der Psychopharmakotherapie in Schwangerschaft und Stillzeit.

Natürlich bietet ein einzelnes Themenheft nicht genug Raum, um alle Aspekte eines so weiten Feldes wie das der schwangerschaftsspezifischen Aspekte neurologischer und psychiatrischer Erkrankungen erschöpfend zu behandeln. Deshalb sei Interessierten die weiterführende Lektüre zweier Sammelbände zu neurologischen Erkrankungen in der Schwangerschaft [3] und zur neurologischen Pharmakotherapie [1] sehr empfohlen.

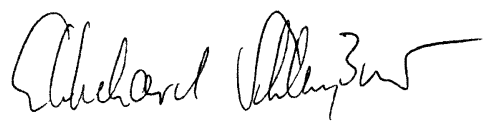

Prof. Dr. Ekkehard Schleußner

\section{Korrespondenzadresse}

\section{Prof. Dr. E. Schleußner}

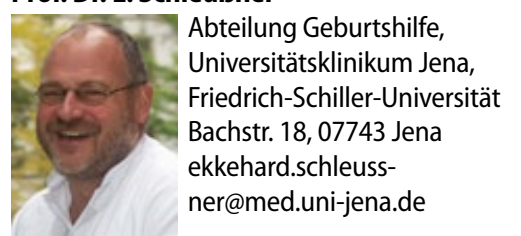

\section{Literatur}

1. Block F (2008) Neurologische Erkrankungen in der Schwangerschaft. In: Block F (Hrsg) Kompendium der neurologischen Pharmakotherapie. Springer, Berlin Heidelberg New York Tokio, S 513-530

2. Schleußner E, Hagemann G, Hunger-Battefeld W (2008) Krampfanfall und Koma bei Schwangeren und Wöchnerinnen. Gynakologe 41: 427-435

3. Berger T, Brezinka C, Luef G (Hrsg) (2007) Neurologische Erkrankungen in der Schwangerschaft. Springer, Berlin Heidelberg New York Tokio

\section{Galenus-von- \\ Pergamon-Preis 2011}

\section{Springer Medizin zeichnet exzellente Forschung und ehrenamtliches Engagement aus}

Auch in diesem Jahr hat Springer Medizin den von der Ärzte Zeitung Verlags $\mathrm{GmbH}$ gestifteten Galenus-von-Pergamon-Preis vergeben und würdigt damit exzellente Forschung in Deutschland. 12 unabhängige Experten haben im Oktober 2011 über die Preisträger in den Kategorien Primary Care, Specialist Care und Grundlagenforschung entschieden. Als Schirmherrin des Galenus-von-PergamonPreises lobte die Bundesministerin für Bildung und Forschung, Frau Professor Dr. Annette Schavan, die herausragenden Leistungen aller Forscher-Teams.

Primary Care

Der Preis in der Kategorie Primary Care würdigt ein Medikament, das bei einer breiten Patientengruppe eingesetzt wird. In diesem Jahr hat Amgen/GlaxoSmithKline (GSK) den Preis für Prolia ${ }^{\oplus}$ erhalten. Mit Prolia ${ }^{\oplus}$ steht erstmals ein monoklonaler Antikörper zur gezielten Osteoporosetherapie zur Verfügung. Der Antiköper hemmt die Knochenresorption und schützt somit Frauen in der Postmenopause und Männer mit Prostatakrebs vor Frakturen.

\section{Specialist Care}

Der Preis in der Kategorie Specialist Care zeichnet ein Medikament aus, das zur Behandlung seltener Erkrankungen verwendet wird. In diesem Jahr ist Amgen der Gewinner für Nplate ${ }^{\oplus}$, das erste zugelassene Medikament zur Stimulierung der Thrombozytenbildung. Es ist indiziert zur Behandlung von Erwachsenen mit einer chronischen Immun-(idiopathischen)thrombozytopenischen Purpura (ITP), die auf andere Therapien nicht ansprechen.
Die Preisträger in diesen beiden Kategorien erhalten jeweils eine Medaille und eine Urkunde.

\section{Grundlagenforschung}

In dieser Kategorie wird ein Bewerber prämiert, der eine herausragende wissenschaftliche Arbeit in der pharmakologischen Grundlagenforschung eingereicht hat. Die Auszeichnung ging an Professor Wolfgang Kühn aus Freiburg. Er und sein Team haben sich in der Forschung um die Entschlüsselung molekularer Mechanismen der autosomal dominanten polyzystischen Nierenerkrankung (ADPKD) verdient gemacht. Der Preisträger erhält zur Medaille und Urkunde zusätzlich ein Preisgeld in Höhe von 10.000 Euro.

\section{CharityAward 2011}

Mit dem im Jahr 2009 erstmals gestifteten CharityAward zeichnet Springer Medizin jährlich Menschen und Organisationen aus, die sich ehrenamtlich um behinderte, kranke und hilfsbedürftige Menschen verdient gemacht haben. Wer Gewinner wird, entscheiden die Leser der Medien von Springer Medizin. Preisträgerin 2011 ist die in Peru geborene Ärztin Jenny De la Torre: Sie wird für ihren inzwischen 16-jährigen Einsatz für Obdachlose in Berlin-Mitte ausgezeichnet. Im Rahmen dieses Engagements hat sie eine Stiftung gegründet und das Berliner "Gesundheitszentrum für Obdachlose" aufgebaut. Der Award besteht aus einem Medienpaket in einem Wert von 100.000 Euro und einem Barscheck über 50.000 Euro. Schirmherr des Preises ist Bundesgesundheitsminister Daniel Bahr. 